\title{
Characterization of patients with myocardial infarction with non-obstructive coronary arteries - experience from the Croatian branch of the ISACS-CT Registry
}

\author{
DInes Vinković1*, \\ DFilip Lončarić ${ }^{2}$, \\ DPetra Mjehović1, \\ DDorja Sabljak ${ }^{3}$, \\ DVedrana Vlahovićc ${ }^{3}$, \\ (D)Gur Salai ${ }^{3}$, \\ (DKlara Klarić ${ }^{3}$, \\ DToni Radić ${ }^{3}$, \\ DSaša Pavasović1, \\ (DNina Jakuš¹, \\ DDora Fabijanović', \\ Dlvo Planinc', \\ (DMaja Čikeš', \\ Davor Miličić'
}

'University of Zagreb School of Medicine, University Hospital Centre Zagreb, Zagreb, Croatia

${ }^{2}$ August Pi i Sunyer Biomedical Research Institute (IDIBAPS), Barcelona, Spain ${ }^{3}$ University of Zagreb School of Medicine, Zagreb, Croatia

RECEIVED:

September 12, 2019

ACCEPTED:

September 16, 2019

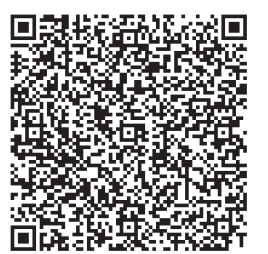

KEYWORDS: acute coronary syndrome, non-obstructive coronary disease.

CITATION: Cardiol Croat. 2019;14(9-10):217-9. | https://doi.org/10.15836/ccar2019.217

*ADDRESS FOR CORRESPONDENCE: Ines Vinković, Klinički bolnički centar Zagreb, Kišpatićeva 12, HR-10000 Zagreb, Croatia. / Phone: +385-91-8905-037 / E-mail: iness.vinkovic@gmail.com

ORCID: Ines Vinković, https://doi.org/0000-0003-1705-8295 • Filip Lončarić, https://doi.org/0000-0002-7865-1108 Petra Mjehović, https://doi.org/0000-0003-4908-4674 • Dorja Sabljak, https://doi.org/0000-0002-7785-5555 Vedrana Vlahović, https://doi.org/0000-0002-8021-4855 • Grgur Salai, https://doi.org/0000-0002-7782-1646 Toni Radić, https://doi.org/0000-0002-2695-3964 • Klara Klarić, https://doi.org/0000-0002-4180-692X

Saša Pavasović, https://doi.org/0000-0002-3705-0226 • Nina Jakuš, https://doi.org/0000-0001-7304-1127 Dora Fabijanović, https://doi.org/0000-0003-2633-3439 • Ivo Planinc, https://doi.org/0000-0003-0561-6704 Maja Čikeš, https://doi.org/0000-0002-4772-5549 • Davor Miličić, https://doi.org/0000-0001-9101-1570

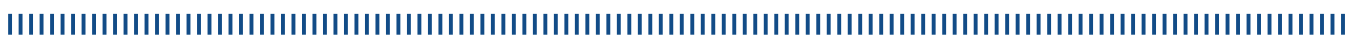

Background and Aim: There are still wide knowledge gaps in myocardial infarction with non-obstructive coronary arteries (MINOCA) - a heterogeneous entity seen in 1-10\% of patients with acute coronary syndrome (ACS) ${ }^{1}$. The aim is to determine characteristics of MINOCA in the Croatian branch of the ISACS-CT registry, and compare them to age- and gender-matched patients with unstable angina (UA), non-ST-segment elevation myocardial infarction (NSTEMI) and ST segment elevation myocardial infarction (STEMI).

Patients and Methods: The study included 2487 patients with ACS. MINOCA was defined by simultaneous cardiac troponin levels $>0.014 \mathrm{ng} / \mathrm{L}$, symptoms of ischemia or significant ST-T changes in the ECG, and an absence of coronary artery stenosis of $\geq 50 \%$ on angiography. Age and gender-matching was performed from the remaining cohort by randomly sampling patients from the UA, STEMI and NSTEMI subgroups, based on the mean age \pm 5 years and the gender ratio of the MINOCA group.

Results: MINOCA was seen in $2.5 \%(n=63)$ of the cohort, initially categorized as UA (37\%), NSTEMI (48\%) and STEMI $(16 \%)$. Median age was $62(53,71)$ years, 56\% male. After age- and gender-matching, there were 36 UA (10\% of the UA cohort), 135 NSTEMI (15\%) and 198 STEMI (16\%) patients in the ACS control subgroups. MINOCA patients had a lower prevalence of diabetes, hypertension, dyslipidemia, chronic kidney disease and tobacco use as compared to UA and NSTEMI. MINOCA patients used less antiplatelets, beta-blockers and statins before hospitalization. MINOCA and STEMI subgroups had a high incidence of chest pain symptoms and a short time from symptom onset to hospitalization. In the first $24 \mathrm{~h}$ of hospitalization, less MINOCA patients were treated with antiplatelets and statins, and at discharge, they were less frequently prescribed with antiplatelet drugs compared to UA and NSTEMI (Table 1). Inhospital mortality was low, with no deaths in MINOCA and UA patients, and 2 and 3 deaths in NSTEMI and STEMI, respectively.

Conclusion: MINOCA patients are mainly categorized as UA and NSTEMI at presentation, but have less comorbidities, more pronounced symptoms of typical chest pain, a shorter time from symptoms to hospitalization, lower levels of statin and antiaggregation prescription at admission, and antiaggregation at discharge. In-hospital mortality confirms MINOCA as low risk, however, long-term registry follow-up is needed to learn about longer term outcomes. 
Characterization of patients with myocardial infarction with non-obstructive coronary arteries - experience from the Croatian branch of the ISACS-CT Registry

TABLE 1. Baseline characteristics and the comparison of patients with myocardial infarction with non-obstructive coronary arteries, unstable angina, ST-segment elevation myocardial infarction and non-ST-segment elevation myocardial infarction.

\begin{tabular}{|c|c|c|c|c|c|c|c|}
\hline & VARIABLES & $\begin{array}{l}\text { MINOCA } \\
(n=63)\end{array}$ & $\begin{array}{c}\text { UA } \\
(n=36)\end{array}$ & $\begin{array}{l}\text { NSTEMI } \\
(n=135)\end{array}$ & $\begin{array}{l}\text { STEMI } \\
(\mathrm{n}=198)\end{array}$ & P-value & Misssing \\
\hline \multirow[t]{3}{*}{ ACS type } & UA & 37 & - & - & - & & 0 \\
\hline & NSTEMI & 48 & - & - & - & & 0 \\
\hline & STEMI & 16 & - & - & - & & 0 \\
\hline \multirow[t]{15}{*}{ General characteristics } & Age & 62 & 60 & 62 & 62 & 0.692 & 0 \\
\hline & Male gender & 56 & 56 & 56 & 56 & 1 & 0 \\
\hline & BMI & 28.7 & 31.2 & 29.3 & 27.7 & 0.001 & 36 \\
\hline & Systolic blood pressure at admission & 145 & 150 & 140 & 140 & 0.003 & 20 \\
\hline & Family history of CAD & 27 & 31 & 31 & 26 & ns & 0 \\
\hline & Diabetes & 18 & 28 & 36 & 23 & 0.017 & 0 \\
\hline & Insulin therapy & 2 & 8 & 11 & 7 & 0.087 & 0 \\
\hline & Hypertension or on th & 77 & 92 & 82 & 76 & 0.118 & 1 \\
\hline & Smoking or ex-smoker & 36 & 56 & 42 & 62 & $<0.001$ & 6 \\
\hline & Hypercholestroleamia or on therapy & 58 & 86 & 69 & 53 & $<0.001$ & 5 \\
\hline & Angina classified by CCS & 7 & 13 & 14 & 5 & 0.057 & 13 \\
\hline & Unstable angina at rest & 8 & 49 & 11 & 2 & $<0.001$ & 8 \\
\hline & Heart failure NYHA 2+ & 5 & 3 & 3 & 2 & 0.412 & 7 \\
\hline & COPD & 2 & 6 & 6 & 7 & 0.539 & 7 \\
\hline & Chronic kidney disease & 5 & 12 & 12 & 4 & 0.028 & 7 \\
\hline \multirow{5}{*}{$\begin{array}{l}\text { Theraphy before admi- } \\
\text { ssion }\end{array}$} & Aspirin & 35 & 43 & 32 & 18 & 0.002 & 6 \\
\hline & Other antiplatlet therapy & 18 & 24 & 10 & 6 & 0.006 & 8 \\
\hline & ACEi & 49 & 57 & 47 & 40 & 0.249 & 6 \\
\hline & Beta-blockers & 44 & 49 & 41 & 22 & $<0.001$ & 6 \\
\hline & Statins & 30 & 39 & 32 & 18 & 0.007 & 6 \\
\hline \multirow[t]{10}{*}{ ACS presentation } & $\begin{array}{l}\text { Two or more } 20 \text { mins chest pain episodes in } \\
\text { the previous } 24 \mathrm{hrs}\end{array}$ & 21 & 48 & 50 & 41 & 0.014 & 33 \\
\hline & Onset $<6$ hours & 63 & 44 & 46 & 70 & $<0.001$ & 3 \\
\hline & Chest pain & 97 & 92 & 90 & 98 & 0.004 & 1 \\
\hline & Abnormal ECG & 46 & 46 & 63 & 99 & $<0.001$ & 1 \\
\hline & LBBB & 4 & 0 & 1 & 5 & 0.514 & 6 \\
\hline & ST elevation & 19 & 6 & 4 & 81 & $<0.001$ & 0 \\
\hline & ST depression & 10 & 14 & 22 & 15 & 0.147 & 0 \\
\hline & Q wave & 5 & 11 & 8 & 15 & 0.074 & 0 \\
\hline & T wave inversion & 3 & 17 & 26 & 10 & $<0.001$ & 0 \\
\hline & Initial descision for PCI treatment & 63 & 83 & 86 & 99 & $<0.001$ & 0 \\
\hline \multirow[t]{8}{*}{ Therapy at admission } & Nitrates & 45 & 57 & 47 & 34 & 0.021 & 10 \\
\hline & Aspirin $24 \mathrm{~h}$ & 100 & 100 & 96 & 96 & 0.394 & 2 \\
\hline & Clopidogrel & 71 & 86 & 90 & 69 & $<0.001$ & 2 \\
\hline & Other antiplatelet therapy & 10 & 9 & 13 & 46 & $<0.001$ & 3 \\
\hline & Statins 24 & 84 & 97 & 95 & 95 & 0.030 & 1 \\
\hline & ACEi 24h & 82 & 86 & 74 & 71 & 0.127 & 3 \\
\hline & Beta blockers $24 \mathrm{~h}$ & 76 & 81 & 75 & 66 & 0.122 & 2 \\
\hline & GP Ilb/IIla & 3 & 12 & 10 & 50 & $<0.001$ & 7 \\
\hline
\end{tabular}


Characterization of patients with myocardial infarction with non-obstructive coronary arteries - experience from the Croatian branch of the ISACS-CT Registry

TABLE 1. Continued.

\begin{tabular}{|c|c|c|c|c|c|c|c|}
\hline & VARIABLES & $\begin{array}{c}\text { MINOCA } \\
(n=63)\end{array}$ & $\begin{array}{c}\text { UA } \\
(n=36)\end{array}$ & $\begin{array}{l}\text { NSTEMI } \\
(\mathrm{n}=135)\end{array}$ & $\begin{array}{c}\text { STEMI } \\
(n=198)\end{array}$ & P-value & Misssing \\
\hline \multirow[t]{14}{*}{ Lab results } & Troponin T & 0.07 & 0.02 & 0.18 & 0.35 & $<0.001$ & 15 \\
\hline & Troponin T peak & 0.2 & 0.04 & 0.7 & 3.64 & $<0.001$ & 25 \\
\hline & CRP & 2 & 38 & 30 & 25 & 0.001 & 35 \\
\hline & CRP-peak & 4 & 39 & 33 & 58 & 0.005 & 52 \\
\hline & Creatinemia & 90 & 88 & 90 & 80 & 0.024 & 5 \\
\hline & WBC & 7.5 & 8.9 & 9.0 & 11.3 & $<0.001$ & 5 \\
\hline & Hemoglobin & 139 & 143 & 140 & 141 & 0.606 & 5 \\
\hline & Cholesterol & 4.7 & 5.1 & 5.2 & 5.0 & 0.419 & 15 \\
\hline & Tryglicerides & 1.28 & 1.61 & 1.63 & 1.40 & 0.001 & 15 \\
\hline & HDL-C & 1.3 & 1.17 & 1.10 & 1.17 & 0.003 & 15 \\
\hline & LDL-C & 2.87 & 3.68 & 3.71 & 3.68 & 0.322 & 15 \\
\hline & Kaliemia & 4.2 & 4.3 & 4.3 & 4.1 & 0.020 & 19 \\
\hline & $\mathrm{Na}$ & 140 & 140 & 140 & 139 & $<0.001$ & 21 \\
\hline & CK peak & 207 & 161 & 165 & 1229 & $<0.001$ & 42 \\
\hline \multirow[t]{10}{*}{ Theraphy at discharge } & Aspirin at discharge & 94 & 100 & 97 & 96 & 0.446 & 0 \\
\hline & Clopidogrel at discharge & 44 & 89 & 87 & 54 & $<0.001$ & 0 \\
\hline & Other antiplatelet therapy at discharge & 0 & 8 & 8 & 47 & $<0.001$ & 1 \\
\hline & Anticoagulants at discharge & 21 & 11 & 7 & 18 & 0.012 & 0 \\
\hline & ACEi at discharge & 81 & 81 & 81 & 81 & ns & 1 \\
\hline & Beta-blockers at discharge & 79 & 81 & 84 & 80 & 0.804 & 0 \\
\hline & Statins at discharge & 89 & 97 & 95 & 94 & 0.360 & 1 \\
\hline & Other lipid lowering drugs at discharge & 3 & 6 & 12 & 8 & 0.202 & 0 \\
\hline & Diuretics at discharge & 24 & 36 & 28 & 27 & 0.624 & 2 \\
\hline & Antiarrhythmics at discharge & 18 & 17 & 12 & 8 & 0.533 & 2 \\
\hline \multirow[t]{3}{*}{ Outcomes } & Duration of hospitalisation & $4(2,5)$ & $3(2,5)$ & $4(3,7)$ & $5(1,7)$ & 0.005 & 0 \\
\hline & EF at discharge & 55 & 60 & 55 & 50 & $<0.001$ & 22 \\
\hline & Hospital mortality & 0 & 0 & 2 & 3 & 0.573 & 0 \\
\hline $\begin{array}{l}\text { MINOCA - Myocardial infar } \\
\text { gment elevation myocardic } \\
\text { - New York Heart Associati } \\
\text { branch block; GP Ilb/IIla - }\end{array}$ & $\begin{array}{l}\text { n with non-obstructive coronary arteries; UA } \\
\text { farction; ACS - Acute coronary syndrome; BM } \\
\text { COPD - Chronic obstructive pulmonary disea } \\
\text { coprotein IIb/IIla; WBC - White blood cells; EF }\end{array}$ & $\begin{array}{l}\text { le angina; ST } \\
\text { nass index; C } \\
\text { Angiotensin } \\
\text { fraction }\end{array}$ & $\begin{array}{l}\text { - ST-segm } \\
\text { - Coronary } \\
\text { nverting-en }\end{array}$ & $\begin{array}{l}\text { elevation my } \\
\text { ery disease; } \\
\text { e inhibitor; }\end{array}$ & $\begin{array}{l}\text { ardial infarc } \\
\text { S - Canadian } \\
\text { G - Electroc }\end{array}$ & $\begin{array}{l}\text {; NSTEMI - } \\
\text { rdiovascula } \\
\text { iogram; LBB }\end{array}$ & $\begin{array}{l}\text { n-ST-se- } \\
\text { ociety; NYHA } \\
\text { Left bundle }\end{array}$ \\
\hline
\end{tabular}

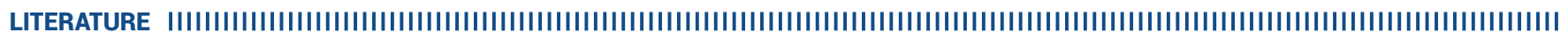

1. Agewall S, Beltrame JF, Reynolds HR, Niessner A, Rosano G, Caforio AL, et al; WG on Cardiovascular Pharmacotherapy. ESC working group position paper on myocardial infarction with nonobstructive coronary arteries. Eur Heart J. 2017 Jan 14;38(3):143-153. https://doi.org/10.1093/eurheartj/ehw149 